\title{
From Biometrics to Forensics: A Feature Collection and First Feature Fusion Approaches for Latent Fingerprint Detection Using a Chromatic White Light (CWL) Sensor
}

\author{
Robert Fischer ${ }^{1}$, Tobias Kiertscher ${ }^{1}$, Stefan Gruhn ${ }^{1}$, \\ Tobias Scheidat ${ }^{1}$, and Claus Vielhauer ${ }^{1,2}$ \\ 1 Brandenburg University of Applied Sciences, Germany \\ \{robert.fischer, tobias.kiertscher, stefan.gruhn, \\ tobias.scheidat, claus.vielhauer\}afh-brandenburg.de \\ 2 Otto-von-Guericke University Magdeburg, Germany
}

\begin{abstract}
Application of non-invasive scan technologies for acquisition of latent fingerprints promise a better support of forensic and dactyloscopic experts when securing evidence at crime scenes. Furthermore, non-destructive acquisition preserve the chance of subsequent chemical and forensic analysis of left residue. Based on results of an ongoing research project with sensor industry partners, this paper presents a collection of 28 statistical, gradient-, and spectral densitybased features for latent fingerprint detection using low resolution scans. Within this work a chromatic white light (CWL) sensor is used for image acquisition. Furthermore, based on concepts of biometric fusion, a taxonomy for possible fusion strategies is presented and very first results for three different strategies on decision level are discussed. Experimental evaluation is performed based on scans of 1680 latent fingerprints on three different surfaces. The results show very good performance on planar, non-absorbing surfaces with uniform reflection characteristics with an detection rate of $2.51 \%$ in the best case. On the other hand difficulties are arising from surfaces with non-uniform/predictable reflection characteristics.
\end{abstract}

\section{Introduction}

Technology-aided support of criminalistic experts, when investigating crime scenes and collecting traces, has become a more and more important application in image acquisition and signal processing. For example manual lifting of latent fingerprints using conventional methods like powder, ninhydrin or cyanoacrylat is time consuming and very limited in its scope of application. New technologies for contact-less and non-invasive acquisition of latent fingerprints promise possibilities to inspect much more and larger surface areas and at the same time might significantly reduce the amount of manual work. Because large area surface scans in high resolution are still very time consuming, there is an urgent need for a reliable detection and localization of latent fingerprints in low resolution scans. This paper presents an approach, partly based on biometric methods, for a semi automated system for acquisition, detection, and localization of latent fingerprints using a chromatic white light (CWL) sensor, which is typically used

B. De Decker and D.W. Chadwick (Eds.): CMS 2012, LNCS 7394, pp. 207-210, 2012.

(C) IFIP International Federation for Information Processing 2012 
in material science and industry. Based on previous work [2 35] new latent fingerprint detection features will be evaluated. Furthermore, certain fusion strategies, which are adopted from biometrics [8] are applied.

\section{Features for Fingerprint Detection}

Statistical Features: For this study we have selected eight out of 40 statistical features we identified in prior work [3]. These features are derived from industrial standards such as ASME B46.1 / ISO 4287/1 / ANSI B.46.1, see [14]. Due to limited space the complete list and explanation of evaluated features can be found in the corresponding poster. Gradient-based Features: As fingerprint locations in gray-scale images are typically characterized by regions with distinct edges, gradient features derived from biometric fingerprint processing might be feasible for fingerprint detection [2]. First feature is coherence evaluation [6] and second feature is basic gradient directional-difference evaluation. Spectral Texture Features: Ridge lines of fingerprints form a pattern in the image with a well bounded frequency range. The frequency range can directly be derived from the typical width of papillary ridges on the finger. [5] is introducing this approach and uses a first selection of a combination from statistical features based on a centered Fourier spectrum. For each of the six spectral features we study three alternative wavebands $(256,512,1024)$ resulting in 18 spectral features.

\section{Fusion Approaches}

To increase the overall performance of biometric systems some approaches for using multi-biometric methods have been suggested in earlier work, for example Jain and Ross [7] classified possible scenarios. Based on these classification and previous work [8] this paper presents possible fusion strategies for a forensic fingerprint detection

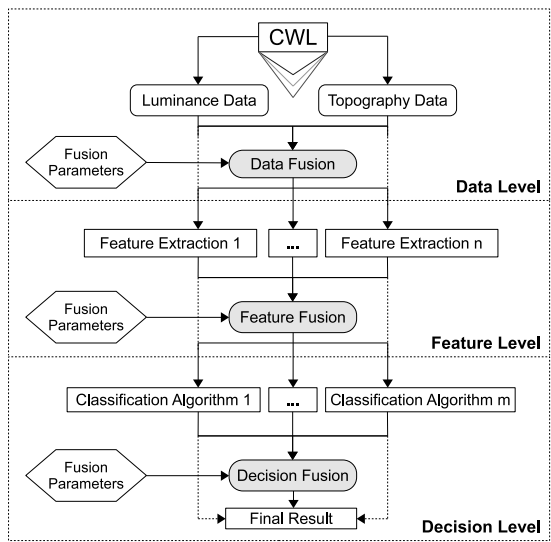

(a) Fusion Level

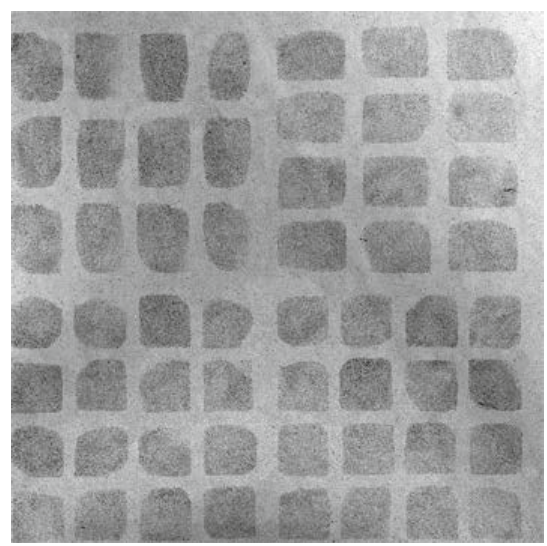

(b) Platter Layout

Fig. 1. a) Illustration fusion level, b) Platter (furniture white) layout with 56 fingerprints 
system. Three different fusion levels and two different fusion strategies known from biometric systems, have been identified for the application in a forensic fingerprint detection system. Data level fusion: The CWL sensor is capable to acquire two different images (topography and luminance) at once. Thus, it might be feasible to fuse these different images on the data level for increased detection performance. This can be charaterized as a multi-sensor system. Though, the luminance and the topography image data is used for the later evaluation, the fusion on data level is not within scope of this paper. Feature level fusion: Since different features have an unequal performance detecting fingerprints on varying surfaces, it might be reasonable to combine results of multiple features for increasing detection performance (see fig. 1a). This can be characterized as a multi-algorithm system. Decision level fusion: It is possible to combine the results of different classification algorithms on decision level, which can also be characterized as a multi-algorithm system. Our first fusion apporaches will focus on decision level. See fig. 1a for a taxonomy of different fusion levels.

\section{Results and Conclusions}

Due to space limitations, our testset summarizes briefly as follows: we are measuring equal error rate (EER) for positive and negative detection of latent fingerprints. Three different surfaces are used: white furniture (FW), brushed metal (BM), and car body finish (NGCB) as representatives for three different difficulty classes (easy, medium, hard). Ten different platters with 56 fingerprints each (see fig. 1 b), have been used for every subtrate leading to a total amount of 1680 fingerprints. There are three main observations, the best EER of $2.51 \%$ is determined for FW using feature SDsd512 with a blocksize of $4 \mathrm{~mm}$. For the other two surfaces the best EERs amount $14.55 \%$ (BM, Smean, blocksize $4 \mathrm{~mm}$ ) and $21.13 \%$ (NGCB, Smean, blocksize $4 \mathrm{~mm}$ ). Complete result tables are presented in the corresponding poster. The differing influence of blocksizes on detection performance based on the three surface groups is the second important observation. While statistical features are most robust features with respect to smaller blocksizes, gradient-based features are more tolerant to smaller blocksizes than spectral density-based ones. Thirdly, it can be observed that weighted decision level fusion leads to only marginal changes compared to single results of individual features. We can state that there are no significant improvements caused by the fusion strategies chosen out of a high number of possibilities. Future work will cover the following aspects: i) surface dependent feature selection, ii) evaluation of additonal fusion strategies, and iii) implementation of fusion on different fusion levels as introduced in section three (see fig. 1. h).

Acknowledgments. This work is supported by the German Federal Ministry of Education and Research (BMBF), project "Digitale Fingerspuren (Digi-Dak)" FKZ: 13N10816. The content of this document is under the sole responsibility of the authors. We would like to thank J. Dittmann, M. Hildebrandt, A. Makrushin, and AMSL research group Magdeburg. 


\section{References}

1. ASME. Surface texture, surface roughness waviness and lay. Technical Report ASME B46.1, American Society of Mechanical Engineers (2009)

2. Fischer, R., Vielhauer, C.: Ausgewaehlte biometrieverfahren zur fingerspurdetektion. In: Proc. of DACH 2011, pp. 374-385 (2011) (German)

3. Gruhn, S., Vielhauer, C.: Surface classification and detection of latent fingerprints: Novel approach based on surface texture parameters. In: Proc. of 7 th Int. Symposium on ISPA, pp. 687-683 (2011)

4. ISO. Geometrical product specifications (gps) - surface texture: Profile method - terms, definitions and surface texture parameters. Technical Report ISO4287, International Organization for Standardization (1997)

5. Kiertscher, T., Fischer, R., Vielhauer, C.: Latent fingerprint detection using a spectral texture feature. In: Proc. of the 13th ACM MMSEC, pp. 27-32. ACM, New York (2011)

6. Maltoni, D., Maio, D., Jain, A.K., Prabhakar, S.: Handbook of Fingerprint Recognition, 2nd edn. Springer (2009)

7. Ross, A., Jain, A.K.: Multimodal biometrics: an overview. In: Proc. of 12th European Signal Processing Conference, pp. 1221-1224 (2004)

8. Scheidat, T., Vielhauer, C., Fischer, R.: Comparative study on fusion strategies for biometric handwriting. In: Proc. of the 13th ACM MMSEC, pp. 61-68. ACM, New York (2011) 